\title{
Diagnosis and management of IgG4- related disease
}

\author{
Vinod S Hegade, ${ }^{1}$ Maria B Sheridan, ${ }^{2}$ Matthew T Huggett $^{1}$
}

${ }^{1}$ Department of

Gastroenterology, St James

University Hospital, Leeds, UK

${ }^{2}$ Department of Radiology, St

James University Hospital, Leeds,

UK

\section{Correspondence to}

Dr Vinod S Hegade, Department of Gastroenterology, St James

University Hospital, Leeds LS9

7TF, UK; vinod.hegade@ncl.ac.uk

Received 8 April 2018

Revised 9 September 2018

Accepted 29 September 2018

Published Online First

31 October 2018

\section{SLinked}

- http://dx.doi.org/10.1136/ flgastro-2019-101192

\section{Check for updates}

(C) Author(s) (or their employer(s)) 2019. No commercial re-use. See rights and permissions. Published by BMJ.

To cite: Hegade VS,

Sheridan MB, Huggett MT.

Frontline Gastroenterology

2019;10:275-283.

\begin{abstract}
IgG subclass 4-related disease (IgG4-RD) is a rare but increasingly recognised fibroinflammatory condition known to affect multiple organs. IgG4-RD is characterised by unique histological features of lymphoplasmacytic infiltration, storiform fibrosis and obliterative phlebitis. In this review we describe the pancreaticobiliary manifestations of IgG4-RD, with particular emphasis on type 1 autoimmune pancreatitis (AIP) and IgG4-related sclerosing cholangitis (IgG4-SC). AIP and IgG4-SC can pose diagnostic challenges to the clinician as they may mimic pancreatic cancer and primary sclerosing cholangitis, respectively. We discuss current knowledge, clinical diagnostic criteria and recent advances and summarise the evidence base for current therapeutic approaches for AIP and IgG4-SC.
\end{abstract}

\section{INTRODUCTION}

Immunoglobulin $G$ subclass 4-related disease (IgG4-RD) is a rare disease characterised by increased serum levels of IgG4 and tissue infiltration by IgG4-positive plasma cells. Since Sarles et al first described a form of idiopathic pancreatitis associated with obstructive jaundice and hypergammaglobulinaemia in $1961{ }^{1}$ the entity has been described under various nomenclatures by different authors (table 1). Most notably, in 1995 Yoshida et al coined the term 'autoimmune pancreatitis' (AIP) to describe a case of a 68-year-old woman with obstructive jaundice, diffusely enlarged pancreas with irregular narrow pancreatic duct, hypergammaglobulinaemia and fibrosis; with subsequent resolution on steroid therapy. ${ }^{2}$ AIP was subsequently recognised as a systemic condition in $2003^{3}$ and later suggested to be the pancreatic manifestation of a systemic disease affecting other organs. ${ }^{4}$

Prior to its official nomenclature of IgG4-RD, various independent studies described the condition affecting different extrapancreatic organs including bile ducts, liver, gastrointestinal tract, lacrimal and salivary glands, central nervous system, thyroid, lung, kidney, prostate, retroperitoneum, skin, breast, arteries and lymph nodes. ${ }^{5}$ Common features in these reports were elevated serum IgG4, histology showing IgG4 positive plasma cells and resolution of illness following steroid therapy. To unify the diseases and link several disorders previously seen as unrelated, a recent consensus recommended the name 'IgG4-RD'. Specific nomenclatures of individual organ manifestation of IgG4-RD have been proposed using 'IgG4-related' as a modifier, except for the pancreatic manifestation for which the recommended term is type 1 AIP (or IgG4-related pancreatitis). ${ }^{6}$ Specific clinical diagnostic criteria for AIP have been proposed by the most recent International Consensus Diagnostic Criteria (ICDC). ${ }^{7}$ Similarly, the preferred nomenclature for IgG4-RD of bile ducts is IgG4-related sclerosing cholangitis (IgG4-SC). IgG4-related hepatopathy refers to liver involvement that is distinct from biliary tract involvement. ${ }^{6}$

In this review, we discuss both AIP and IgG4-SC from a clinician's perspective, focusing on the clinical characteristics, diagnosis and treatment.

\section{OVERVIEW OF IGG4-RD}

IgG4-RD is a rare entity with an estimated incidence of 60 per million/0.28-1.08 per 100000 population. ${ }^{8}$ An epidemiological study from Japan showed that the overall prevalence of AIP was 2.2/100 000 population, with an annual incidence rate of $0.9 / 100000$ population. ${ }^{9}$ Typically, IgG4-RD (and type 1 AIP) occurs most commonly in middle-aged and older men. The cause of IgG4-RD is unclear and the precise mechanism involved in the pathogenesis of IgG4-SC and AIP remains enigmatic. Current evidence suggests that 
Table 1 Previous nomenclatures used for lgG4-related disease (adapted from Okazaki et al [5])

\begin{tabular}{ll}
\hline Year & Name \\
\hline 1892 & Mikulicz's disease \\
1967 & Familial multifocal fibrosclerosis \\
1972 & Küttner tumour \\
1991 & Lymphoplasmacytic sclerosing pancreatitis \\
1995 & Autoimmune pancreatitis \\
\hline 2006 & IgG4-related sclerosing disease \\
2006 & IgG4-related plasmacytic disease \\
\hline 2008 & IgG4-multiorgan lymphoproliferative syndrome \\
\hline
\end{tabular}

IgG4-RD is an immune-mediated process but target autoantigen(s) and the effector cells have not been fully identified. Recently, annexin A11, an intracellular protein, has been identified as a novel autoantigen in AIP and IgG4-SC. ${ }^{10}$ A potential role of exposure to occupational antigens has been suggested by cohort studies that observed a higher prevalence of the disease among 'blue-collar workers' (eg, building contractors, plumbers). For example, in a Dutch study of 25 patients with IgG4-SC and/or AIP, $88 \%$ had a history of blue-collar work of at least 1 year. ${ }^{11}$ Similarly, in the Oxford cohort of 44 patients with established IgG4$\mathrm{RD}, 61 \%$ of the patients had blue-collar professions and recalled chronic exposures to potentially harmful compounds. ${ }^{11}$ These findings suggest a potential role of chronic exposure to occupational hazards such as solvents, industrial and metal dusts; and pigments and oils used in the automotive industry. This, in addition to the previously observed clonal expansions of IgG4switched $\mathrm{B}$ cells in patients with $\operatorname{IgG} 4-\mathrm{RD},{ }^{12}$ suggests an occupational antigen-driven immune process in the initiation and/or maintenance of IgG4-RD in susceptible individuals.

\section{Clinical features}

IgG4-RD has a varied clinical presentation but usually presents subacutely. Often the disorder is identified incidentally through radiological findings or unexpectedly in pathological specimens. ${ }^{13}$ Symptoms are usually mild and include the presence of painless swellings and mass lesions. Clinical symptoms vary depending on the organ in which the lesions are located. In a prospective study of 115 patients with AIP/IgG4-SC, 74\% had jaundice at presentation, 37\% described a history of abdominal pain and $56 \%$ of patients with type 1 AIP also had IgG4-SC. ${ }^{14}$ Thirty-six per cent of patients had disease beyond the pancreas or biliary tree with diffuse lymphadenopathy as the most common manifestation. ${ }^{14}$ Other extrapancreaticobiliary manifestations of IgG4-RD included sialadenitis, renal infiltrates or masses, pulmonary disease with nodules, interstitial lung disease, or pulmonary fibrosis, ocular manifestations and neurological sequelae including hypopituitarism caused by a pituitary mass and a rapidly progressive autoimmune encephalitis. ${ }^{14}$ Untreated IgG4-SC can lead to hepatic failure within months. ${ }^{15}$ Therefore, a high index of suspicion is warranted to diagnose IgG4-RD since severe organ damage and even death can result if IgG4-RD is left untreated.

In IgG4-RD, the imaging appearances vary considerably and features are generally non-specific. In AIP, pancreatic abnormalities on imaging can be seen in up to $85 \%$ of cases with a diffusely enlarged 'sausageshaped' pancreas (43\%), and a discrete pancreatic mass with or without diffuse enlargement (54\%) as the most common findings. ${ }^{14}$ The presence of a peripancreatic halo is due to a fibroinflammatory process extending into peripancreatic adipose tissue; diffuse narrowing of the pancreatic duct corresponds to non-occlusive periductal inflammation. ${ }^{16}$

\section{Serology}

Elevated serum IgG4 levels have been associated with IgG4-RD but an elevated serum IgG4 is not specific for the disease. Interestingly, serum IgG4 is normal in up to $40 \%$ of patients with biopsy-proven IgG4-RD. ${ }^{17}$ In a recent large study, serum IgG4 was found to be elevated in $82 \%$ of patients with IgG4-RD, but only $22 \%$ of those with an elevated IgG4 met IgG4-RD diagnostic criteria. ${ }^{18}$ In this study, an IgG4 cut-off of $1.4 \mathrm{~g} / \mathrm{L}$ had a sensitivity of $82.8 \%$ and specificity of $84.7 \%$ to diagnose IgG4-RD and a cut-off value $\geq 2.8 \mathrm{~g} / \mathrm{L}$ was useful in distinguishing between IgG4-RD and non-IgG4-RD diagnoses and predicting multiorgan involvement. ${ }^{18}$

\section{Histology}

Histology is critical for the diagnosis of IgG4-RD irrespective of the organ involvement. The three major histopathological features associated with IgG4-RD include dense lymphoplasmacytic infiltrate, fibrosis (arranged at least focally in a storiform pattern) and obliterative phlebitis. ${ }^{19}$ In glandular organs the infiltrate tends to aggregate around ductal structures and the inflammatory lesions which arise result in massforming tissue destructive lesions. ${ }^{19}$ Mild to moderate eosinophil infiltrates are also a key morphologic feature, but neutrophils are detected only rarely and granulomas are distinctly unusual. ${ }^{13}$ The presence of IgG4 positive (IgG4+) plasma cells on immunohistochemical staining is required for the diagnosis of IgG4-RD but clinical correlation is essential. Since IgG4+ cells are found in a wide variety of inflammatory infiltrates, the detection of substantial numbers of IgG4+ plasma cells is therefore not diagnostic of IgG4-RD. ${ }^{13}$ To distinguish from other conditions, semiquantitative analysis with variety of cut-off points has been proposed, including $\geq 10$ to $>50$ IgG4 + plasma cells per high-power field (hpf) or a ratio of IgG4-bearing plasma cells to IgG-bearing plasma cells $>50 \% .^{2021}$ 


\section{Diagnostic criteria for IgG4-RD}

The diagnosis of IgG4-RD is based on a combination of clinical, biochemical, radiological and histological findings. As noted above, histology is critical for the diagnosis and the international histological consensus criteria proposed IgG4+/IgG + plasma cell ratio of $>40 \%$ as mandatory for histological diagnosis of IgG4-RD. ${ }^{22}$ In addition, in the liver, bile duct and pancreas $>10$ IgG4 + cells/hpf by biopsy and $>50$ IgG4 + cells/hpf by surgical specimen are needed. ${ }^{22}$ Using comprehensive diagnostic criteria combined with organ-specific criteria, a diagnosis of IgG4-RD is definite in patients who fulfil the criteria: (1) organ enlargement, mass or nodular lesions, or organ dysfunction; (2) a serum $\operatorname{IgG} 4$ concentration $>1.35 \mathrm{~g} / \mathrm{L}$, and (3) histopathological findings of $>10 \mathrm{IgG} 4+$ cells/hpf and an IgG4/IgG cell ratio $>40 \%$.

\section{TYPE 1 AIP (IGG4-RELATED PANCREATITIS)}

The ICDC for AIP has proposed two distinctive types of AIP type 1 and type 2 (comprehensively reviewed in ref 23). Type I AIP is classically a disease of older men $(>80 \%$ are men) with $>80 \%$ of patients being over the age of 50years. ${ }^{24-26}$ Patients with type 1 AIP most commonly present with obstructive jaundice and minimal abdominal pain with pancreatic enlargement mimicking pancreatic cancer.

Serological abnormalities include elevated titres of IgG and its subset IgG4. Although elevated serum IgG4 is the single best marker of AIP it is not pathognomonic or diagnostic of AIP. Serum IgG4 level in AIP has variable sensitivity (67\%-94\%) and specificity (89\%$100 \%$ ) with a low positive predictive value, deeming the test on its own not suitable for diagnosis. ${ }^{27-29}$ Serum IgG4 level cut-off value of $>134 \mathrm{mg} / \mathrm{dL}$ was shown to have sensitivity and specificity $>90 \% .{ }^{30}$ It is noteworthy that $7 \%-10 \%$ of patients with pancreatic cancer $^{27} 31$ and $>10 \%$ with cholangiocarcinoma (CCA) have elevated serum IgG4.$^{23}$ Serum amylase and lipase are usually normal or mildly elevated in AIP. ${ }^{32}$

Histology is the gold standard for the diagnosis, subtyping into type 1 or type 2 (see below) and differentiating from pancreatic cancer. Endoscopic ultrasound with fine needle aspiration or biopsy (EUS-FNA or EUS-FNB) is the preferred modality for issue acquisition. ${ }^{33}$ Histologically, type 1 AIP is termed as lymphoplasmacytic sclerosing pancreatitis with characteristic features that include ${ }^{54-36}:$ (1) abundant infiltration of plasma cells (>10 IgG4 + cells per hpf and an IgG4 to IgG ratio $>40 \%$ ) and lymphocytes; (2) peculiar storiform or swirling fibrosis; and (3) perivenular infiltration with lymphocytes and plasma cells often leading to obliterative phlebitis.

Type 2 AIP is more common among Western population, accounting for 14\% of AIP compared with 4\% of AIP in Asian countries. ${ }^{37}$ It is rare and shows important differences from type 1 AIP that include: younger age of presentation (mean 40 years), ${ }^{37}$ no serum IgG4 or IgG elevation, absence of autoantibodies and no systemic/ other organ involvement. Therefore, type 2 AIP is not a part of the IgG4-RD spectrum. ${ }^{38}$ The characteristic histology of type 2 AIP is termed non-alcoholic duct-destructive pancreatitis, idiopathic duct-centric pancreatitis or granulocyte epithelial lesion-positive pancreatitis. $^{39}$

\section{Diagnostic criteria of AIP}

Over the years, a number of clinical diagnostic criteria have been proposed to diagnose type 1 AIP. $^{40-43}$ The most familiar is the Mayo Clinic (HISORt) criteria (2006) which include ${ }^{26}$ : characteristic Histology; typical pancreatic/other Imaging; Serology (IgG4>140 mg/dL or $0.86 \mathrm{~g} / \mathrm{L}$ ); Other organ involvement and Response to steroid treatment. In 2009, revised HISORt criteria were proposed to differentiate AIP from pancreatic cancer. ${ }^{44}$ Most recent (2011) guidelines from the ICDC are based on the consensus opinion of the International Association of Pancreatology and propose that diagnosis of type 1 AIP requires a combination of five primary cardinal features ${ }^{7}:(1)$ imaging features of pancreatic parenchyma and pancreatic duct, (2) serology (IgG4 level), (3) other organ involvement, (4) histology of the pancreas, and (5) response to steroid treatment (table 2). Each criterion (except for steroid responsiveness) is classified as either level 1 or level 2 collateral criteria. Level 1 collateral is highly suggestive of AIP. The diagnostic algorithm proposed by the ICDC has been validated and among the five major criteria for AIP the ICDC criteria were found to be the most sensitive (95.1\%). ${ }^{45}$

\section{Differentiation from pancreatic cancer}

Both AIP and pancreatic cancer present with obstructive jaundice and a mass in the pancreas, and the differentiation based on clinical features alone is challenging. Imaging needs to be carefully reviewed and it is recommended that AIP should be considered only after negative workup for pancreatic malignancy. ${ }^{7}$ Typical imaging findings (on CT or MRI) in AIP are diffuse enlargement of pancreas with delayed enhancement and long ( $>1 / 3$ length of the main pancreatic duct) or multiple strictures without marked upstream dilatation $^{7}$ (figure 1). On the contrary, typical findings of pancreatic cancer are a low-density mass on contrast-enhanced CT, and pancreatic ductal dilatation/ cut-off with or without pancreatic atrophy. However, some patients with AIP may show low-density masses, pancreatic ductal dilatation or distal atrophy. Such imaging findings in patients with obstructive jaundice and/or a pancreatic mass are highly suggestive of pancreatic cancer and should be managed as pancreatic cancer. ${ }^{7}$

\section{IGG4-RELATED SCLEROSING CHOLANGITIS}

IgG4-SC is a biliary disease of unknown immunopathogenesis with cholangiographic features 
Table 2 The International Consensus of Diagnostic Criteria (ICDC) for definite and probable type 1 autoimmune pancreatitis (adapted from Shimosegawa et al [7])

\begin{tabular}{|c|c|c|c|}
\hline Criterion & \multicolumn{2}{|l|}{ Level 1} & Level 2 \\
\hline $\begin{array}{l}\text { P. Parenchymal } \\
\text { imaging }\end{array}$ & \multicolumn{2}{|c|}{$\begin{array}{l}\text { Typical: } \\
\text { Diffuse enlargement with delayed enhancement (sometimes associated } \\
\text { with rim-like enhancement) }\end{array}$} & $\begin{array}{l}\text { Indeterminate (including atypical): } \\
\text { Segmental/focal enlargement with delayed enhancement }\end{array}$ \\
\hline $\begin{array}{l}\text { D. Ductal imaging } \\
\text { (ERP) }\end{array}$ & \multicolumn{2}{|c|}{$\begin{array}{l}\text { Long (>1/3 length of the main pancreatic duct) or multiple strictures } \\
\text { without marked upstream dilatation }\end{array}$} & $\begin{array}{l}\text { Segmental/focal narrowing without marked upstream } \\
\text { dilatation (duct size }<5 \mathrm{~mm} \text { ) }\end{array}$ \\
\hline S. Serology & \multicolumn{2}{|c|}{$\lg G 4>2 \times$ upper limit of normal value } & IgG4 1-2x upper limit of normal value \\
\hline \multirow{3}{*}{$\begin{array}{l}\text { 00I. Other organ } \\
\text { involvement }\end{array}$} & \multicolumn{2}{|c|}{$A$ or $B$} & $A$ or $B$ \\
\hline & \multicolumn{2}{|c|}{$\begin{array}{l}\text { A. Histology of extrapancreatic organs } \\
\text { Any three of the following: } \\
\text { 1. Marked lymphoplasmacytic infiltration with fibrosis and without } \\
\text { granulocytic infiltration. } \\
\text { 2. Storiform fibrosis. } \\
\text { 3. Obliterative phlebitis. } \\
\text { 4. Abundant (>10 cells/hpf) lgG4 positive cells. }\end{array}$} & $\begin{array}{l}\text { A. Histology of extrapancreatic organs including } \\
\text { endoscopic biopsies of bile duct*} \\
\text { Both of the following: } \\
\text { 1. Marked lymphoplasmacytic infiltration without } \\
\text { granulocytic infiltration. } \\
\text { 2. Abundant (>10 cells/hpf) IgG4 positive cells. }\end{array}$ \\
\hline & \multicolumn{2}{|c|}{$\begin{array}{l}\text { B. Typical radiological evidence } \\
\text { At least one: } \\
\text { 1. Segmental/multiple proximal (hilar/intrahepatic) or proximal and } \\
\text { distal bile duct stricture. } \\
\text { 2. Retroperitoneal fibrosis. }\end{array}$} & $\begin{array}{l}\text { B. Physical or radiological evidence } \\
\text { At least one: } \\
\text { 1. Symmetrically enlarged salivary/lacrimal glands. } \\
\text { 2. Radiologic evidence of renal involvement described } \\
\text { in association with AIP. }\end{array}$ \\
\hline $\begin{array}{l}\text { H. Histology of } \\
\text { pancreas }\end{array}$ & \multicolumn{2}{|c|}{$\begin{array}{l}\text { LPSP (core biopsy/resection) } \\
\text { At least three of the following: } \\
\text { 1. Periductal lymphoplasmacytic infiltrate without granulocytic } \\
\text { infiltration. } \\
\text { 2. Obliterative phlebitis. } \\
\text { 3. Storiform fibrosis. } \\
\text { 4. Abundant (>10 cells/hpf) IgG4 positive cells. }\end{array}$} & $\begin{array}{l}\text { LPSP (core biopsy) } \\
\text { Any two of the following: } \\
\text { 1. Periductal lymphoplasmacytic infiltrate without } \\
\text { granulocytic infiltration. } \\
\text { 2. Obliterative phlebitis. } \\
\text { 3. Storiform fibrosis. } \\
\text { 4. Abundant (>10 cells/hpf) IgG4 positive cells. }\end{array}$ \\
\hline $\begin{array}{l}\text { Rt. Response to } \\
\text { steroidt }\end{array}$ & \multicolumn{3}{|c|}{$\begin{array}{l}\text { Diagnostic steroid trial } \\
\text { Rapid ( } \leq 2 \text { weeks) radiologically demonstrable resolution or marked improvement in pancreatic/extrapancreatic manifestations }\end{array}$} \\
\hline Diagnosis & \multicolumn{2}{|l|}{$\begin{array}{l}\text { Primary basis for } \\
\text { diagnosis }\end{array}$} & Collateral evidence \\
\hline \multirow[t]{4}{*}{ Definite type 1 AlP } & Histology & Typical/indeterminate & \\
\hline & \multirow[t]{2}{*}{ Imaging } & Typical & Any non-D level 1/level 2 \\
\hline & & Indeterminate & Two or more from level 1 (+level $2 \mathrm{D} \ddagger$ ) \\
\hline & Response to steroid & Indeterminate & Level $1 \mathrm{~S} / \mathrm{OOI}+$ Rt or level $1 \mathrm{D}+$ level $2 \mathrm{~S} / 00 \mathrm{I} / \mathrm{H}+\mathrm{Rt}$ \\
\hline Probable type 1 AlP & & Indeterminate & Level $2 \mathrm{~S} / 0 \mathrm{OI} / \mathrm{H}+\mathrm{Rt}$ \\
\hline
\end{tabular}

*Endoscopic biopsy of duodenal papilla is a useful adjunctive method because ampulla is often involved pathologically in AIP.

†Diagnostic steroid trial should be conducted carefully by pancreatologists with caveats only after negative workup for cancer including endoscopic ultrasound with fine needle aspiration (EUS-FNA).

fLevel $2 \mathrm{D}$ is counted as level 1 in this setting.

AIP, autoimmune pancreatitis; ERP, endoscopic retrograde pancreatography; hpf, high-power field; LPSP, lymphoplasmacytic sclerosing pancreatitis.

indistinguishable from primary sclerosing cholangitis (PSC), but distinct histological findings and a dramatic response to corticosteroids (CS). ${ }^{46}$ It is a common extrapancreatic manifestation of IgG4-RD characterised by increased serum IgG4 levels and presence of dense infiltration of IgG4+ plasma cells with storiform fibrosis and/or obliterative phlebitis in the bile duct wall. ${ }^{47} 48$

In the Mayo Clinic cohort of 53 patients with IgG4SC, median age at diagnosis was 60 years in a predominantly male patient group (85\%), frequently presenting with obstructive jaundice. ${ }^{49}$ In IgG4-SC, typical biliary tract imaging reveals diffuse or segmental narrowing of the intrahepatic and/or extrahepatic bile duct associated with thickening of the bile duct wall. ${ }^{50} \mathrm{Clin}$ ical and radiological features of IgG4-SC mimic those of PSC and hilar CCA, and both must be rigorously excluded before making diagnosis of IgG4-SC. ${ }^{51}$ In a Dutch case series of 185 patients who had undergone resection on the suspicion of hilar CCA, two (1.1\%) had features of IgG4-SC. ${ }^{52}$

Differentiating IgG4-SC from PSC and CCA can be challenging since biliary tract biopsy is difficult and sufficient biliary tract tissue may not be available to determine the characteristic histology of IgG4SC. Referral to a specialist Hepato-Pancreato-Biliary (HPB) centre may be needed for comprehensive diagnostic evaluation including endoscopic biliary biopsy 
A

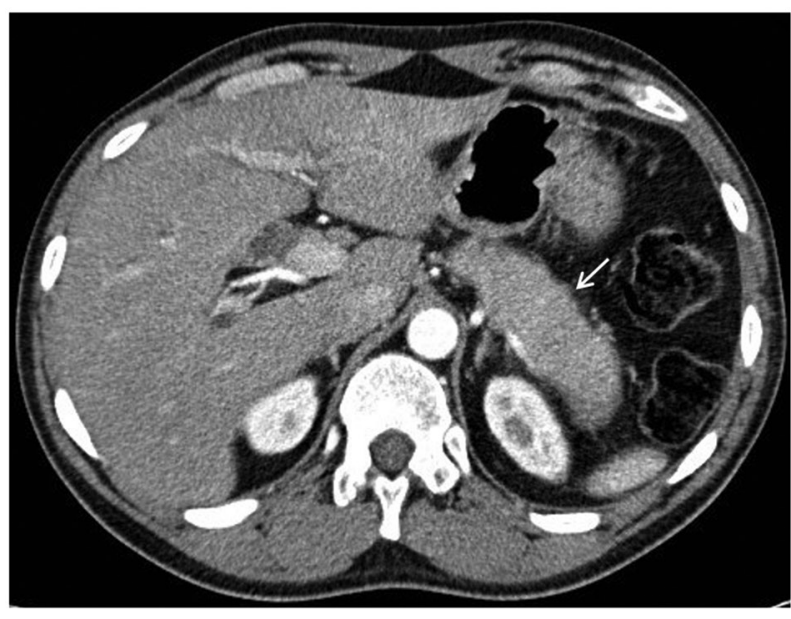

B

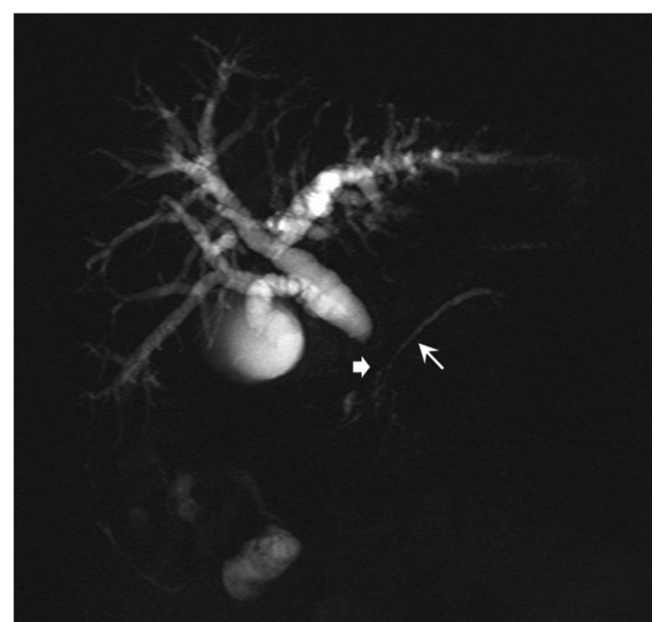

Figure 1 Radiological features of autoimmune pancreatitis. (A) CT abdomen image showing diffuse enlargement of the pancreas with loss of its lobular contour and presence of a peripancreatic rim/halo (arrow). (B) MR cholangiopancreatography (MRCP) image of the same patient showing distal common bile duct stricture (arrowhead) and narrowing of the main pancreatic duct without upstream dilatation (arrow).

and EUS-FNA/B. ${ }^{50}$ Lack of association with inflammatory bowel disease (IBD) is an important feature differentiating IgG4-SC from PSC, although IgG4+ plasma cells have been detected in colonic specimens in patients with AIP and IgG4-related colitis mimicking IBD. ${ }^{53}$ Interestingly, approximately $9 \%$ of previously diagnosed patients with PSC had elevated serum IgG4 levels which was associated with more severe disease course and more aggressive course before and after liver transplantation. 5556

Similar to AIP, diagnostic criteria for IgG4-SC have been proposed and are based on the combination of the following criteria ${ }^{50}:$ (1) characteristic biliary imaging findings; (2) elevation of serum IgG4 concentrations; (3) coexistence of IgG4-RDs except those of the biliary tract; and (4) characteristic histopathological features. Effectiveness of steroid therapy is an optional additional diagnostic criterion to confirm an accurate diagnosis of IgG4-SC. ${ }^{50}$

\section{Treatment}

Once diagnosis of IgG4-RD is confirmed, CS treatment is the recommended first-line therapy (unless there is a major contraindication for CS use). Indeed, dramatic response to CS is a hallmark of IgG4-RD irrespective of additional organ involvement. There have been no prospective randomised controlled studies of optimal treatment regimens and the goals of treatment with CS therapy are not defined. Nevertheless, in clinical practice, CS treatment has been shown to result in improvement in symptoms (including resolution of jaundice) and liver biochemistry, with reduction of serum IgG4 levels and reversal of biliary strictures on cholangiogram in patients with type 1 AIP and IgG4-SC. ${ }^{149}$

The CS treatment protocol in expert centres is to give $0.5 \mathrm{mg} / \mathrm{kg}$ of prednisolone $(30-40 \mathrm{mg} /$ day) for 2-4 weeks and then reduce the dose by $5 \mathrm{mg}$ per
1-2 weeks, with regular monitoring of biochemistry and repeat imaging after week 4 (figure 2), with an expected cessation of treatment by $4-6$ months. ${ }^{14}$ In patients with diabetes or deemed more likely to get steroid-induced complications, a lower dose of $20 \mathrm{mg}$ prednisolone can be used.

By initial CS induction treatment, remission can be successfully induced in almost all subjects with type 1 (and type 2) AIP. For instance, in their large, multicentre international study, Hart et al showed 99\% of patients with type 1 AIP treated with CS achieved clinical remission. ${ }^{37}$ In addition to CS therapy, endoscopic (endoscopic retrograde cholangiopancreatography (ERCP)) biliary stenting may be indicated to relieve obstructive jaundice. In such cases, biliary stenting is usually temporary and may be removed in the majority of patients in 2-4 weeks during the first course of steroid therapy. ${ }^{1423}$

\section{Relapse and its treatment}

Relapse during steroid taper or following withdrawal of steroids is common and has been documented in $40 \%$ of patients with IgG4-SC after completing an 11-week course of steroids. ${ }^{49}$ In another study, poststeroid relapse rate was $50 \%$ at a median time of 4.6 months after stopping the first course of steroids. ${ }^{14}$ In the Hart et al's study of 1064 patients with AIP, relapses typically occurred in the pancreas or biliary tree and were more common in patients with type $1(31 \%)$ versus type 2 $(9 \%, \mathrm{p}<0.001)$ AIP. Factors predicting relapse are not entirely clear but IgG4-SC has been shown as an important predictor of relapse with higher relapse rates $(56 \%$ vs $26 \%, \mathrm{p}<0.001) .{ }^{14} 37$ In addition, strictures of the proximal extrahepatic and intrahepatic bile ducts are associated with a higher risk of relapse compared with those with strictures of the distal ducts. ${ }^{49}$ 
A

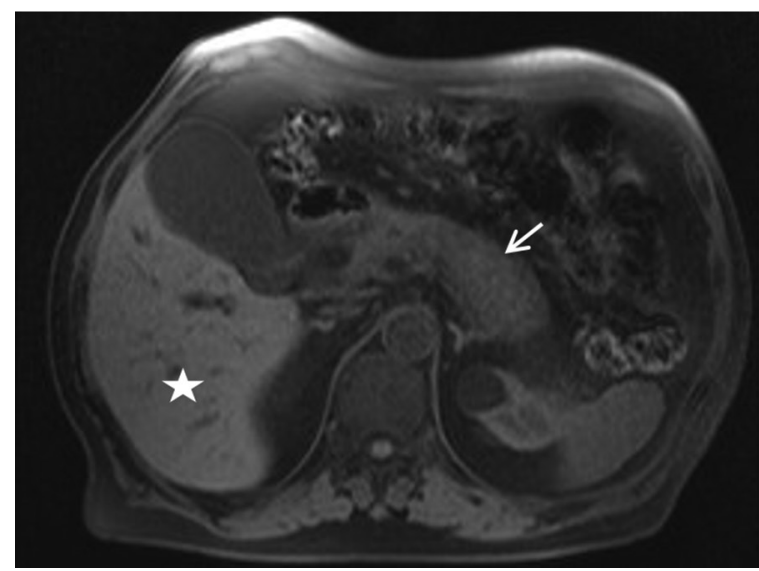

B

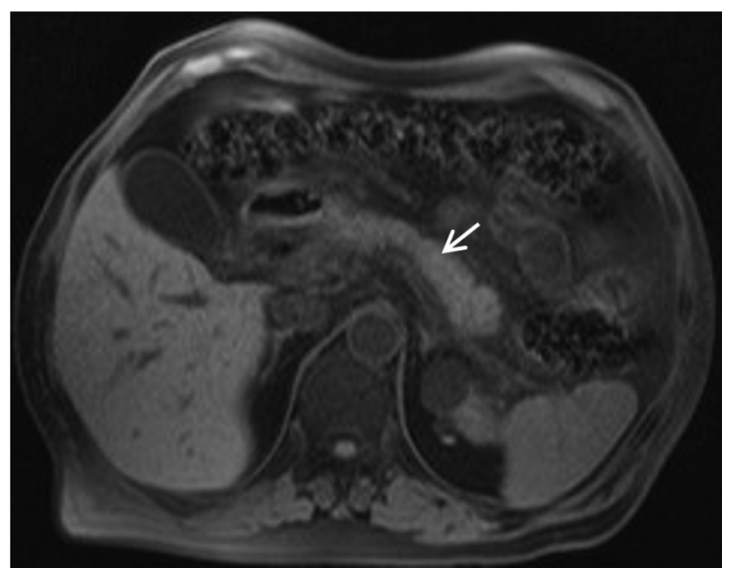

Figure 2 Radiological changes in autoimmune pancreatitis (AIP), presteroid and poststeroid treatment. (A) T1-weighted fat suppressed MRI image showing diffusely low signal intensity (SI) from pancreas (arrow) (normally SI from pancreas is same or higher than SI from liver, marked as*). (B) Four weeks after steroid treatment pancreas volume is decreased (arrow) but $\mathrm{SI}$ is returning to normal.

Previously, raised serum IgG4 at diagnosis, lack of decline in serum IgG4 and normalisation of serum IgG4 levels with treatment have not been shown to predict relapse. ${ }^{14} 57$ However, a recent large study observed that serum IgG4 of $\geq 2.8 \mathrm{~g} / \mathrm{L}$ at diagnosis was associated with increased risk of relapse in IgG4-RD. ${ }^{18}$

Since an IgE-mediated allergic response appears to develop in most patients with IgG4-RD, it has been suggested that levels of IgE might be used in predicting relapse. In this regard, a recent prospective study of 48 patients with IgG4-RD showed a serum IgE level $>380 \mathrm{kIU} / \mathrm{L}$ at diagnosis identified patients with disease relapse with $88 \%$ specificity, $64 \%$ sensitivity and a likelihood ratio of 5.4. ${ }^{58}$ However, these findings need external validation before routine use of serum IgE levels can be recommended for predicting relapse.

There are currently no international guidelines for the treatment of relapse in IgG4-RD. Relapse should be treated with repeat courses of steroid therapy, and/ or additional second-line immunosuppressive therapy (eg, azathioprine at doses up to $2 \mathrm{mg} / \mathrm{kg} / \mathrm{day}$ ). Mycophenolate mofetil (500-1000 mg two times per day) or methotrexate ( $15 \mathrm{mg}$ weekly) or mercaptopurine can be used as alternative immunosuppression in the event of azathioprine intolerance or side effects.

\section{Treatment of refractory disease}

In patients with IgG4-RD refractory to CS or other immunosuppressive agents, rituximab, a monoclonal antibody directed against CD20 antigen on B lymphocytes, is a therapeutic option. ${ }^{59-61}$ There are no controlled trials of rituximab and the current evidence is based on observational studies using rituximab in patients with IgG4-RD refractory to steroid or standard immunosuppressive drugs. The recommended dosage is $1 \mathrm{~g}$ intravenous infusion administered on day 1 and repeated on day 15 .
The collective evidence suggests rituximab is clinically effective with almost all study participants having clinical and serological responses (rapid decline in IgG4) enabling withdrawal and discontinuation of CS and other immunosuppressive drugs. Following rituximab treatment complete remissions were sustained for at least 6 months in $\sim 50 \%$ of patients, with $40 \%$ having a disease response for a year. ${ }^{59}$ Relapse in responders is common with baseline elevations in serum IgG4, IgE and blood eosinophil concentrations shown to predict IgG4-RD relapses independently. ${ }^{62}$

Repeated rituximab courses have been shown to maintain their effectiveness and result in further decreases in IgG4 concentrations, better disease control and quiescent disease. ${ }^{60}$ A recent French study on long-term efficacy and safety of rituximab in 33 patients with IgG4-RD suggests maintenance therapy with systematic rituximab infusions (ie, before occurrence of a relapse) is associated with longer relapsefree survival. ${ }^{63}$

The recent National Institute for Health and Care Excellence guidance (published in December 2016) recommends rituximab as a third-line therapy for a small number of highly selected patients with IgG4-RD to control disease and prevent further progression to fibrosis and organ damage. ${ }^{64}$ The eligible patient group is relapsed patients with active disease that is no longer controlled with conventional therapies who either fail to respond to primary treatment or with adverse reactions or contraindications to CS plus azathioprine or methotrexate or mycophenolate mofetil. ${ }^{64}$

\section{PROGNOSIS AND CANCER RISK}

Limited data are available on the long-term sequelae in patients with AIP and IgG4-SC. Pancreatic exocrine insufficiency (53\%) and type 2 diabetes (37\%) are common ${ }^{14}$ and other reported non-malignant 
complications include pancreatic duct stones, ${ }^{37}$ renal impairment and portal and/or splenic vein thrombosis (without variceal bleeding). ${ }^{14}$

In IgG4-RD multiple organs are affected in $60 \%-90 \%$ of patients ${ }^{65}$ and the disease can be associated with significant morbidity and mortality owing to extrapancreatic organ failure and malignancy. In a recent study, mortality rate was $10 \%$ during follow-up with an increased OR for risk of death $(2.07,95 \%$ CI 1.07 to $3.55, \mathrm{p}=0.02$ ) compared with matched national statistics. ${ }^{14}$

Evidence suggests an increased risk of cancers in patients with type 1 AIP during follow-up; most frequently occurring cancers were gastric, lung and prostate, with most cancers diagnosed $>3$ years after AIP diagnosis. ${ }^{37}$ In a UK study, $6 \%$ of patients with AIP and/or IgG4-SC were diagnosed with a malignancy during a median follow-up of 33 months with an incidence ratio for all cancers of 2.25 (95\% CI 1.23 to $3.94, p=0.02$ ) compared with the general population. ${ }^{14}$ In a multicentre, retrospective cohort study, AIP was associated with an increased risk of pancreatic cancer in the first year after diagnosis ${ }^{66}$ and another Japanese study reported annual incident rate of pancreatic cancer in type 1 AIP to be $0.92 \% .{ }^{67}$ Despite the reported association of pancreatic cancer in type 1 AIP, the role of surveillance during follow-up is not clear.

\section{CONCLUSION}

IgG4-RD is an increasingly recognised, immune-mediated, multiorgan, systemic disease with type 1 AIP and IgG4-SC as its pancreatic and biliary manifestations, respectively. Significant progress has been made in the clinical characterisation of IgG4-RD but much remains unknown about its aetiology and pathogenesis. For the accurate diagnosis of AIP and IgG4-SC it is paramount that clinical features are carefully correlated with imaging appearances, histological architecture and immunohistochemical stains. To aid clinicians and pathologists, comprehensive diagnostic criteria are available to diagnose AIP and differentiate the condition from pancreatic cancer, its close mimic. CS are the mainstay of treatment with a remarkable response rate. Further research is needed for more detailed understanding of IgG4-RD, including the role of IgG4 in the disease mechanisms, identification of more specific disease markers, predictors of response to treatment and data on long-term outcomes.

Contributors VSH drafted the main manuscript. MBS contributed in writing the radiology sections and images. MTH provided intellectual inputs and revised the draft. All authors read and approved the final manuscript.

Funding The authors have not declared a specific grant for this research from any funding agency in the public, commercial or not-for-profit sectors.

Competing interests None declared.

Patient consent Not required.
Provenance and peer review Not commissioned; internally peer reviewed.

\section{REFERENCES}

1 Sarles H, Sarles JC, Muratore R, et al. Chronic inflammatory sclerosis of the pancreas-an autonomous pancreatic disease? Am J Dig Dis 1961;6:688-98.

2 Yoshida K, Toki F, Takeuchi T, et al. Chronic pancreatitis caused by an autoimmune abnormality. Proposal of the concept of autoimmune pancreatitis. Dig Dis Sci 1995;40:1561-8.

3 Kamisawa T, Egawa N, Nakajima H. Autoimmune pancreatitis is a systemic autoimmune disease. Am J Gastroenterol 2003;98:2811-2.

4 Chari ST, Murray JA. Autoimmune pancreatitis, Part II: the relapse. Gastroenterology 2008;134:625-8.

5 Okazaki K, Uchida K, Ikeura T, et al. Current concept and diagnosis of IgG4-related disease in the hepato-bilio-pancreatic system. J Gastroenterol 2013;48:303-14.

6 Stone JH, Khosroshahi A, Deshpande V, et al. Recommendations for the nomenclature of IgG4-related disease and its individual organ system manifestations. Arthritis Rheum 2012;64:3061-7.

7 Shimosegawa T, Chari ST, Frulloni L, et al. International consensus diagnostic criteria for autoimmune pancreatitis: guidelines of the International Association of Pancreatology. Pancreas 2011;40:352-8.

8 Uchida K, Masamune A, Shimosegawa T, et al. Prevalence of IgG4-Related Disease in Japan Based on Nationwide Survey in 2009. Int J Rheumatol 2012;2012:1-5.

9 Kanno A, Nishimori I, Masamune A, et al. Nationwide epidemiological survey of autoimmune pancreatitis in Japan. Pancreas 2012;41:835-9.

10 Hubers LM, Vos H, Schuurman AR, et al. Annexin A11 is targeted by IgG4 and IgG1 autoantibodies in IgG4-related disease. Gut 2018;67:728-35.

11 de Buy Wenniger LJ, Culver EL, Beuers U. Exposure to occupational antigens might predispose to IgG4-related disease. Hepatology 2014;60:1453-4.

12 Maillette de Buy Wenniger LJ, Doorenspleet ME, Klarenbeek PL, et al. Immunoglobulin G4+ clones identified by nextgeneration sequencing dominate the $\mathrm{B}$ cell receptor repertoire in immunoglobulin G4 associated cholangitis. Hepatology 2013;57:2390-8.

13 Stone JH, Zen Y, Deshpande V. IgG4-related disease. N Engl J Med 2012;366:539-51.

14 Huggett MT, Culver EL, Kumar M, et al. Type 1 autoimmune pancreatitis and IgG4-related sclerosing cholangitis is associated with extrapancreatic organ failure, malignancy, and mortality in a prospective UK cohort. Am J Gastroenterol 2014;109:1675-83.

15 Björnsson E. Immunoglobulin G4-associated cholangitis. Curr Opin Gastroenterol 2008;24:389-94.

16 Sahani DV, Kalva SP, Farrell J, et al. Autoimmune pancreatitis: imaging features. Radiology 2004;233:345-52.

17 Sah RP, Chari ST. Serologic issues in IgG4-related systemic disease and autoimmune pancreatitis. Curr Opin Rheumatol 2011;23:108-13.

18 Culver EL, Sadler R, Simpson D, et al. Elevated serum IgG4 levels in diagnosis, treatment response, organ involvement, and relapse in a prospective IgG4-related disease UK cohort. Am J Gastroenterol 2016;111:733-43.

19 Deshpande V, Gupta R, Sainani N, et al. Subclassification of autoimmune pancreatitis: a histologic classification with clinical significance. Am J Surg Pathol 2011;35:26-35.

20 Dhall D, Suriawinata AA, Tang LH, et al. Use of immunohistochemistry for IgG4 in the distinction of autoimmune pancreatitis from peritumoral pancreatitis. Hum Pathol 2010;41:643-52. 
21 Deshpande V, Chicano S, Chiocca S, et al. Autoimmune pancreatitis: a systemic immune complex mediated disease. Am J Surg Pathol 2006;30:1537-45.

22 Deshpande V, Zen Y, Chan JK, et al. Consensus statement on the pathology of IgG4-related disease. Mod Pathol 2012;25:1181-92.

23 Park DH, Kim MH, Chari ST. Recent advances in autoimmune pancreatitis. Gut 2009;58:1680-9.

24 Kamisawa T, Egawa N, Nakajima H, et al. Clinical difficulties in the differentiation of autoimmune pancreatitis and pancreatic carcinoma. Am J Gastroenterol 2003;98:2694-9.

25 Church NI, Pereira SP, Deheragoda MG, et al. Autoimmune pancreatitis: clinical and radiological features and objective response to steroid therapy in a UK series. Am J Gastroenterol 2007;102:2417-25.

26 Chari ST, Smyrk TC, Levy MJ, et al. Diagnosis of autoimmune pancreatitis: the Mayo Clinic experience. Clin Gastroenterol Hepatol 2006;4:1010-6. quiz 934.

27 Ghazale A, Chari ST, Smyrk TC, et al. Value of serum IgG4 in the diagnosis of autoimmune pancreatitis and in distinguishing it from pancreatic cancer. Am J Gastroenterol 2007;102:164653

28 Choi EK, Kim MH, Lee TY, et al. The sensitivity and specificity of serum immunoglobulin G and immunoglobulin G4 levels in the diagnosis of autoimmune chronic pancreatitis: Korean experience. Pancreas 2007;35:156-61.

29 Morselli-Labate AM, Pezzilli R. Usefulness of serum IgG4 in the diagnosis and follow up of autoimmune pancreatitis: a systematic literature review and meta-analysis. J Gastroenterol Hepatol 2009;24:15-36.

30 Hamano H, Kawa S, Horiuchi A, et al. High serum IgG4 concentrations in patients with sclerosing pancreatitis. N Engl J Med 2001;344:732-8.

31 Raina A, Krasinskas AM, Greer JB, et al. Serum immunoglobulin $G$ fraction 4 levels in pancreatic cancer: elevations not associated with autoimmune pancreatitis. Arch Pathol Lab Med 2008;132:48-53.

32 Chintanaboina J, Yang Z, Mathew A. Autoimmune pancreatitis: a diagnostic challenge for the clinician. South Med J 2015;108:579-89.

33 Iwashita T, Yasuda I, Doi S, et al. Use of samples from endoscopic ultrasound-guided 19-gauge fine-needle aspiration in diagnosis of autoimmune pancreatitis. Clin Gastroenterol Hepatol 2012;10:316-22.

34 Kawaguchi K, Koike M, Tsuruta K, et al. Lymphoplasmacytic sclerosing pancreatitis with cholangitis: a variant of primary sclerosing cholangitis extensively involving pancreas. Hum Pathol 1991;22:387-95.

35 Kamisawa T. IgG4-positive plasma cells specifically infiltrate various organs in autoimmune pancreatitis. Pancreas 2004;29:167-8.

36 Zhang L, Notohara K, Levy MJ, et al. IgG4-positive plasma cell infiltration in the diagnosis of autoimmune pancreatitis. Mod Pathol 2007;20:23-8.

37 Hart PA, Kamisawa T, Brugge WR, et al. Long-term outcomes of autoimmune pancreatitis: a multicentre, international analysis. Gut 2013;62:1771-6.

38 Chari ST, Kloeppel G, Zhang L, et al. Histopathologic and clinical subtypes of autoimmune pancreatitis: the Honolulu consensus document. Pancreas 2010;39:549-54.

39 Notohara K, Burgart LJ, Yadav D, et al. Idiopathic chronic pancreatitis with periductal lymphoplasmacytic infiltration clinicopathologic features of 35 cases. Am J Surg Pathol 2003;27:1119-27.

40 Hardt P. Diagnostic criteria for autoimmune pancreatitis by the Japan Pancreas Society. J Jpn Pan Soc 2002;17:585-7.

41 Okazaki K, Kawa S, Kamisawa T, et al. Clinical diagnostic criteria of autoimmune pancreatitis: revised proposal. J Gastroenterol 2006;41:626-31.
42 Kwon S, Kim MH, Choi EK. The diagnostic criteria for autoimmune chronic pancreatitis: it is time to make a consensus. Pancreas 2007;34:279-86.

43 Otsuki M, Chung JB, Okazaki K, et al. Asian diagnostic criteria for autoimmune pancreatitis: consensus of the Japan-Korea Symposium on Autoimmune Pancreatitis. J Gastroenterol 2008;43:403-8.

44 Chari ST, Takahashi N, Levy MJ, et al. A diagnostic strategy to distinguish autoimmune pancreatitis from pancreatic cancer. Clin Gastroenterol Hepatol 2009;7:1097-103.

45 Sumimoto K, Uchida K, Mitsuyama T, et al. A proposal of a diagnostic algorithm with validation of International Consensus Diagnostic Criteria for autoimmune pancreatitis in a Japanese cohort. Pancreatology 2013;13:230-7.

46 Björnsson E, Chari ST, Smyrk TC, et al. Immunoglobulin G4 associated cholangitis: description of an emerging clinical entity based on review of the literature. Hepatology 2007;45:1547-54

47 Zen Y, Harada K, Sasaki M, et al. IgG4-related sclerosing cholangitis with and without hepatic inflammatory pseudotumor, and sclerosing pancreatitis-associated sclerosing cholangitis: do they belong to a spectrum of sclerosing pancreatitis? Am J Surg Pathol 2004;28:1193-203.

48 Okazaki K, Uchida K, Koyabu M, et al. IgG4 cholangiopathy: current concept, diagnosis, and pathogenesis. J Hepatol 2014;61:690-5.

49 Ghazale A, Chari ST, Zhang L, et al. Immunoglobulin G4associated cholangitis: clinical profile and response to therapy. Gastroenterology 2008;134:706-15.

50 Ohara $\mathrm{H}$, Okazaki $\mathrm{K}$, Tsubouchi $\mathrm{H}$, et al. Clinical diagnostic criteria of IgG4-related sclerosing cholangitis 2012. J Hepatobiliary Pancreat Sci 2012;19:536-42.

51 Oh HC, Kim MH, Lee KT, et al. Clinical clues to suspicion of IgG4-associated sclerosing cholangitis disguised as primary sclerosing cholangitis or hilar cholangiocarcinoma. J Gastroenterol Hepatol 2010;25:1831-7.

52 Erdogan D, Kloek JJ, ten Kate FJ, et al. Immunoglobulin G4-related sclerosing cholangitis in patients resected for presumed malignant bile duct strictures. Br J Surg 2008;95:727-34.

53 Narula N, Vasudev M, Marshall JK. IgG4-related sclerosing disease: a novel mimic of inflammatory bowel disease. Dig Dis Sci 2010;55:3047-51.

54 Dastis SN, Latinne D, Sempoux C, et al. Ulcerative colitis associated with IgG4 cholangitis: similar features in two HLA identical siblings. J Hepatol 2009;51:601-5.

55 Zhang L, Lewis JT, Abraham SC, et al. IgG4+ plasma cell infiltrates in liver explants with primary sclerosing cholangitis. Am J Surg Pathol 2010;34:88-94.

56 Mendes FD, Jorgensen R, Keach J, et al. Elevated serum IgG4 concentration in patients with primary sclerosing cholangitis. Am J Gastroenterol 2006;101:2070-5.

57 Frulloni L, Scattolini C, Falconi M, et al. Autoimmune pancreatitis: differences between the focal and diffuse forms in 87 patients. Am J Gastroenterol 2009;104:2288-94.

58 Culver EL, Sadler R, Bateman AC, et al. Increases in IgE, eosinophils, and mast cells can be used in diagnosis and to predict relapse of IgG4-related disease. Clin Gastroenterol Hepatol 2017;15:1444-52.

59 Carruthers MN, Topazian MD, Khosroshahi A, et al. Rituximab for IgG4-related disease: a prospective, open-label trial. Ann Rheum Dis 2015;74:1171-7.

60 Khosroshahi A, Carruthers MN, Deshpande V, et al. Rituximab for the treatment of IgG4-related disease: lessons from 10 consecutive patients. Medicine 2012;91:57-66.

61 Topazian M, Witzig TE, Smyrk TC, et al. Rituximab therapy for refractory biliary strictures in immunoglobulin G4-associated cholangitis. Clin Gastroenterol Hepatol 2008;6:364-6. 
62 Wallace ZS, Mattoo H, Mahajan VS, et al. Predictors of disease relapse in IgG4-related disease following rituximab. Rheumatology 2016;55:1000-8.

63 Ebbo M, Grados A, Samson M, et al. Long-term efficacy and safety of rituximab in IgG4-related disease: Data from a French nationwide study of thirty-three patients. PLoS One 2017;12:e0183844.

64 NHS England. Clinical commissioning policy: rituximab for immunoglobulin G4-related disease (IgG4-RD). London: Reference: NHS England: 16057/P, 2016.
65 Stone JH, Brito-Zerón P, Bosch X, et al. Diagnostic approach to the complexity of IgG4-related disease. Mayo Clin Proc 2015;90:927-39.

66 Shiokawa M, Kodama Y, Yoshimura K, et al. Risk of cancer in patients with autoimmune pancreatitis. Am J Gastroenterol 2013;108:610-7.

67 Ikeura T, Miyoshi H, Uchida K, et al. Relationship between autoimmune pancreatitis and pancreatic cancer: a single-center experience. Pancreatology 2014;14:373-9. 